\title{
Solution of a Well-Field Design Problem with Implicit Filtering
}

\author{
K. R. Kavanagh, C. T. Kelley \\ North Carolina State University, Center for Research in Scientific Computation and \\ Department of Mathematics, Box 8205, Raleigh, N. C. 27695-8205, USA \\ (krkavana@unity.ncsu.edu,Tim_Kelley@ncsu.edu). \\ C. T. Miller \\ Center for the Advanced Study of the Environment, Department of Environmental Sciences \\ and Engineering, University of North Carolina, Chapel Hill, North Carolina 27599-7431, \\ USA(casey_miller@unc.edu).
}

C. E. Kees, R. M. Darwin, J. P. Reese

North Carolina State University, Center for Research in Scientific Computation and

Department of Mathematics, Box 8205, Raleigh, N. C. 27695-8205, USA

(chris_kees@ncsu.edu,rwdarwin@unity.ncsu.edu,jpreese@unity.ncsu.edu).

\section{W. Farthing}

Center for the Advanced Study of the Environment, Department of Environmental Sciences and Engineering, University of North Carolina, Chapel Hill, North Carolina 27599-7431, USA (matthew_farthing@unc.edu).

\section{S. C. Reed}

North Carolina Supercomputing Center 3021 Cornwallis Rd. Research Triangle Park, NC 27709, USA (reed@ncsc.org).

\begin{abstract}
Problems involving the management of groundwater resources occur routinely, and management decisions based upon optimization approaches offer the potential to save substantial amounts of money. However, this class of application is notoriously difficult to solve due to non-convex objective functions with multiple local minima and both nonlinear models and nonlinear constraints. We solve a subset of community test problems from this application field using MODFLOW, a standard groundwater flow model, and IFFCO, an implicit filtering algorithm that was designed to solve problems similar to those of focus in this work. While sampling methods have received only scant attention in the groundwater optimization literature, we show encouraging results that suggest they are deserving of more widespread consideration for this class of problems. In keeping with our objectives for the community problems, we have packaged the approaches used in this work to facilitate additional work on these problems by others and the application of implicit filtering to other problems in this field. We provide the data for our formulation and solution on the web.
\end{abstract}

Keywords: Implicit filtering, Well field design, Groundwater flow and transport

\section{Introduction}

Groundwater resources are important because about $50 \%$ of the population of the United States rely upon this resource for drinking water. Typical goals in groundwater resources management include designing and managing systems to supply drinking water and to restore contaminated drinking water to 
a usable quality at a minimum cost. Accomplishing these goals requires a model to describe the system of concern, an appropriate objective function, constraints, and an optimization algorithm. The objective function and constraints provide the linkage between the simulation model and the optimizer. Because porous media systems are typically heterogeneous over small scales and described by nonlinear processes, subsurface simulators can be expensive to evaluate and subject to uncertainty, or stochastic in nature. The resulting optimization problems can also be diffi cult, with objective functions that are non-convex and have multiple local minima, and both models and constraints that are nonlinear. For these reasons, subsurface optimization problems are both important and challenging.

To aid the evolution of optimal design of subsurface fbw and transport applications, a set of community problems (CP's) was developed, [30], that are typical of problems commonly encountered and which cover a range of complexity. It was reasoned that focusing on a common set of CP's would allow for not only advancement of approaches to solve an important set of problems, but also a means to aid comparison of various aspects of the solution approach on the same set of problems. It was also anticipated that the CP's would catalyze the introduction of new classes of optimization methods into the groundwater fi eld and result in more active participation of the applied mathematics community in the evolution of solution approaches for this class of application.

A subset of the CP's is a standard water supply application. Roughly speaking, the objective is to locate a set of water supply production wells and fi nd their pumping rates such that cost is minimized subject to constraints on the total amount of water that must be produced, the hydraulic head in the wells, the production capacity of a well, and the portion of the domain over which a well may be located. Evaluation of the objective function requires a groundwater fbw simulator that solves for hydraulic head as a function of space and time given a spatial and temporal domain, material properties, auxiliary conditions, and well design information.

Common approaches for solving water supply management problems include gradient-based methods or genetic algorithm (GA) approaches in which a candidate set of well locations is selected and the pumping rate of each well is a design variable. Gradient-based methods are not reliable because of the non-convex, noisy nature of the problem. Global optimization methods such as genetic and evolutionary algorithms [38, 40, 24, 1], simulated annealing [28, 39], and tabu search [45] have been applied to many subsurface remediation problems (see [30] for many more references). However, these methods can be expensive. Sampling methods are a potentially attractive alternative class of approach for this sort of problem, which have received scant attention in the water resources community but have been concluded to be deserving of a more thorough investigation. 
In this paper we take the view that there is enough structure in the problem to use a deterministic sampling method. These methods are designed to solve problems with diffi cult, but not violently oscillatory optimization landscapes, such as the ones in Figures 8, 9, 10, 11 in $\S 8$. Gradient-based methods are likely to have trouble with such problems, either fi nding local minima, stagnating, or failing to fi nd a descent direction. In our testing of a gradient-based method, which we report in $\S 8$, we observed this type of failure. The NelderMead [34], Hooke-Jeeves [23], MDS [16, 43], DIRECT [25], and implicit fi ltering $[21,20,26]$ are examples of discrete sampling methods.

The objectives of this work are: (1) to provide an initial analysis of a subset of groundwater CP's, which have been recently published; (2) to formulate a solution to these problems with a sampling method, in this case implicit fi ltering; (3) to compare the results with a genetic algorithm approach, and to explain why traditional gradient-based methods can and do fail; (4) to examine the characteristics of the solution space and illustrate the challenges that this class of problem poses; and (5) to point the way toward future improvements for the solution of this class of problem.

\section{Conceptual Model}

An aquifer is a fully saturated, water-bearing region and is considered confi ned if bounded on both the top and the bottom by essentially impermeable material. An unconfi ned aquifer has the water table as its upper bound. The main difference between the two geological formations is that the saturated thickness of an unconfi ned aquifer varies as the hydraulic head varies, thus leading to a nonlinear free-boundary problem.

We consider a well-fi eld design problem. The hydrological settings are homogeneous confi ned and unconfi ned aquifers in three spatial dimensions. For the problems considered, a set of wells is distributed in the domain. Each well is allowed either to inject or extract water. Well-fi eld design problems involve the selection of well locations and pumping rates to minimize the cost of water production. The cost of supplying water typically involves the cost to drill, equip, and connect wells to a treatment or distribution system, and the cost to pump the water and maintain the well. In turn, the cost to pump groundwater depends upon the energy needed to lift the water from its level below the ground surface to the discharge point and to supply suffi cient discharge pressure to achieve the desired fbw.

The decision variables for this type of problem are the pumping rates $\left\{Q_{i}\right\}_{i=1}^{n}\left(\mathrm{~m}^{3} / \mathrm{s}\right)$ at the $n$ wells in the model and the locations $\left\{\left(x_{i}, y_{i}\right)\right\}_{i=1}^{n}$ of the wells. Pumping rates can be constant or variable in time depending upon the application. In the application considered in this paper, a constant 
fbw rate is realistic. This is because any transients decay very early in the fi ve year time horizon.

\section{Formulation}

The physical domain, see Figure 1 , is $\Omega=[0,1000] \times[0,1000] \times[0,30] \mathrm{m}$ with the ground elevation at $z_{g s}=60 \mathrm{~m}$ for the confi ned aquifer and $z_{\mathrm{gs}}=30$ $\mathrm{m}$ for the unconfi ned aquifer.

Flow in saturated porous media can be described, [30], by

$$
S_{s} \frac{\partial h}{\partial t}=\nabla \cdot(K \nabla h)+\mathcal{S},
$$

where $S_{s}(1 / \mathrm{m})$ is the specifi c storage coeffi cient, the unknown $h(\mathrm{~m})$ is the hydraulic head, $K(\mathrm{~m} / \mathrm{s})$ is the hydraulic conductivity [30]. Here the source term $\mathcal{S}$ is a model of the wells, a sum of $\delta$-functions that satisfi es

$$
\int_{\Omega} \mathcal{S}(t) d \Omega=\sum_{i=1}^{n} Q_{i} .
$$

$\Omega$ is the spatial domain. The wells are assumed to extract from near the bottom of the aquifer. If a numerical solution is discrete in the $z$-dimension then only the bottom layer of cells/elements should include the well source terms.

For the confi ned aquifer, we use the following boundary and initial conditions:

$$
\begin{gathered}
\left.\frac{\partial h}{\partial x}\right|_{x=0}=\left.\frac{\partial h}{\partial y}\right|_{y=0}=\left.\frac{\partial h}{\partial z}\right|_{z=0}=0, t>0 \\
q_{z}(x, y, 30, t>0)=-1.903 \times 10^{-8}(\mathrm{~m} / \mathrm{s}) \\
h(1000, y, z, t>0)=50-0.001 y(\mathrm{~m}) \\
h(x, 1000, z, t>0)=50-0.001 x(\mathrm{~m}) \\
h(x, y, z, 0)=h_{s}
\end{gathered}
$$

Here

$$
q_{z}=-K \frac{\partial h}{\partial z}
$$

is the Darcy flux out of the domain, a negative sign in eqn (4) thus represents fbw into the aquifer or recharge that could be the result of rainfall infi ltration or leakage from an overlying aquifer, and $h_{s}$ is the steady state solution to the 
fbw problem prior to the addition of wells. We use $S_{s}=10^{-6}(1 / \mathrm{m})$. For the unconfi ned aquifer, (4), (5) and (6) are replaced with

$$
\begin{gathered}
q_{z}(x, y, h, t>0)=-1.903 \times 10^{-8}(\mathrm{~m} / \mathrm{s}), \\
h(1000, y, z, t>0)=20-0.001 y(\mathrm{~m}),
\end{gathered}
$$

and

$$
h(x, 1000, z, t>0)=20-0.001 x(\mathrm{~m}) .
$$

$S_{s}=2.0 \times 10^{-1}$ is the specifi c yield of the unconfi ned aquifer. For the homogeneous applications, $K=5.01 \times 10^{-5}(\mathrm{~m} / \mathrm{s})$.

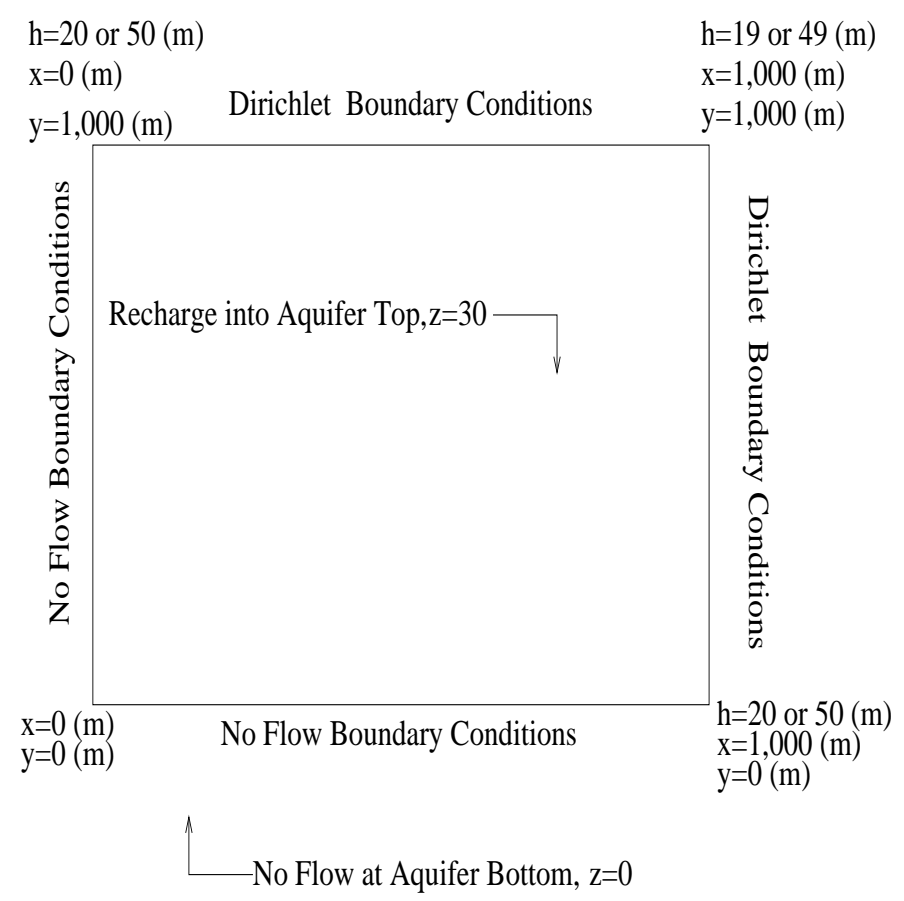

Figure 1. Physical Domain

\section{Objective Function}

We consider a capital cost $f^{c}$ and an operational cost $f^{o}$ seeking to minimize $f^{T}=f^{c}+f^{o}$. The objective function depends on the pumping rates $\left\{Q_{i}\right\}_{i=1}^{n}$ and locations $\left\{\left(x_{i}, y_{i}\right)\right\}_{i=1}^{n}$ of $n$ operating wells. Note that $Q_{i}<0$ means the well is extracting water, and $Q_{i}>0$ means the well is injecting water. For this work, we begin with a virtual fi xed well fi eld containing $N$ wells with the number of operating wells $n \leq N$. 
Since there is a fi xed installation cost for wells, an important aspect of the optimization procedure is the manner in which wells are removed from the design, thereby signifi cantly decreasing the total cost. A well is considered installed and operating if $\left|Q_{i}\right|>0.0001$. If the optimizer specifi es a value with $\left|Q_{i}\right| \leq 0.0001$ then we neither apply the well source term nor do we include the cost of the well in the objective function. This approach results in non-smoothness in the objective function, but provides a reasonable mechanism for removing wells from the design that our optimizer was capable of triggering.

The objective function is given by

$$
\begin{aligned}
& f^{T}=\underbrace{\sum_{i=1}^{n} c_{0} d_{i}^{b 0}+\sum_{Q_{i}<-.0001} c_{1}\left|Q_{i}^{m}\right|^{b_{1}}\left(z_{g s}-h^{m i n}\right)^{b_{2}}}_{f^{c}}+ \\
& \underbrace{t_{f}\left(\sum_{i, Q_{i}<-.0001} c_{2} Q_{i}\left(h_{i}-z_{g s}\right)+\sum_{i, Q_{i}>.0001} c_{3} Q_{i}\right)}_{f^{\circ}},
\end{aligned}
$$

where the cost coeffi cients $c_{j}$ and exponents $b_{j}$ are given in Table I. Here $d_{i}$ is the depth of well $i, Q_{i}^{m}$ is the design pumping rate, $h^{\text {min }}$ is the minimum allowable head, $h_{i}$ is the hydraulic head in well $i, t_{f}$ is the total design time, which was taken as 5 years, and $z_{g s}$ is the elevation of the ground surface. Injection wells are assumed to operate under gravity feed. In $f^{c}$, the fi rst term denotes the cost to install all the wells, and the second term accounts for the additional cost for pumps for the extraction wells. In $f^{o}$ we have a lift cost that applies to the extraction wells and an injection cost that applies to the injection wells.

The design pumping rates $\left\{Q_{i}^{m}\right\}$, i.e. the maximum rates at which a given well can pump, depend upon the aquifer properties, casing and discharge piping size, pump characteristics, screen length and opening size, effectiveness of the development, and local geochemical conditions. One could, in principal, treat the properties of the wells and pumps as optimization parameters. We do not do this here, and focus on more fundamental aspects of the formulation.

\section{Constraints}

We constrain the hydraulic head and pumping rates for the objective function given in (11). The constraints are given by 
Table I. Objective Function Data

\begin{tabular}{|c|c|c|}
\hline data & value & units \\
\hline$c_{0}$ & $5.5 \times 10^{3}$ & $\$ / \mathrm{m}^{b}$ \\
$c_{1}$ & $5.75 \times 10^{3}$ & $\$ /\left[\left(\mathrm{m}^{3} / \mathrm{s}\right)^{b_{1}} \cdot \mathrm{m}^{b_{2}}\right]$ \\
$c_{2}$ & $2.90 \times 10^{-4}$ & $\$ / \mathrm{m}^{4}$ \\
$c_{3}$ & $1.45 \times 10^{-4}$ & $\$ / \mathrm{m}^{3}$ \\
$b_{0}$ & 0.3 & - \\
$b_{1}$ & 0.45 & - \\
$b_{2}$ & 0.64 & - \\
$z_{g s}$ & 60 confined & $m$ \\
$z_{g s}$ & 30 unconfined & $m$ \\
$d_{i}$ & $z_{g s}$ & $m$ \\
$Q_{i}^{m}$ & $1.5 Q_{i}$ & $\mathrm{~m}^{3} / \mathrm{s}$ \\
\hline
\end{tabular}

$$
\begin{gathered}
Q_{T}=\sum_{i=1}^{n} Q_{i} \leq Q_{T}^{\text {min }}, \\
Q^{\text {emax }} \leq Q_{i} \leq Q^{\text {imax }}, i=1, \ldots, n,
\end{gathered}
$$

and

$$
h^{\min } \leq h_{i} \leq h^{\max }, i=1, \ldots, n,
$$

where $Q_{T}$ is the net pumping rate, $Q_{T}^{\min }$ is minimum allowable total extraction rate, $Q^{\text {emax }}$ is the maximum extraction rate at any well, $Q^{i m a x}$ is the maximum injection rate at any well, $h^{\max }$ is the maximum allowable head, and $h^{\text {min }}$ is the minimum allowable head. Values for the bounds in the constraints are given in Table II. We require that the wells be at least $200 \mathrm{~m}$ from the boundary on which Dirichlet boundary conditions are applied, i. e.

$$
0 \leq x_{i}, y_{i} \leq 800 \text {. }
$$

In addition to (15), we do not allow two wells to occupy the same grid point. In the course of the optimization, if two wells converge to the same location, our choice of simulator would implement the two wells as one well, operating at the sum of the two pumping rates. In turn, only one well would operate in the fbw simulation, yet two wells would be included in the installation cost. For our choice of spatial discretization, this indirectly implies that the distance between wells is at least $20 \mathrm{~m}$ apart.

Constraint (12) sets a minimum target for extraction, which is the purpose of the well fi eld. For the problem considered here, the installation costs are far 
more that the operating costs for a single year. Therefore, once the minimum extraction target is reached, it would only make sense to drill additional wells if the long-term operating savings is signifi cant. Since fi ve wells extracting at the maximum level satisfy (12) with equality, one logical formulation of the problem is to fi nd the optimal location of fi ve wells, each extracting as much as possible.

Constraint (13) refects physical limits on the pumps and well design. Well designs are typically limited by the size distribution of the porous medium and the resulting size of the well screen.

The upper bound in constraint (14) keeps the hydraulic head below the surface elevation and the lower bound ensures that excessive drawdown will not occur. This constraint is a linear function of the pumping rates for the confi ned case but a nonlinear function for the unconfi ned case, and in both cases a highly nonlinear function of the locations of the wells.

Table II. Constraint Data

\begin{tabular}{|c|c|c|}
\hline data & value & units \\
\hline$Q_{T}^{\text {min }}$ & $-3.2 \times 10^{-2}$ & $\mathrm{~m}^{3} / \mathrm{s}$ \\
$Q^{\text {emax }}$ & $-6.4 \times 10^{-3}$ & $\mathrm{~m}^{3} / \mathrm{s}$ \\
$Q^{\text {imax }}$ & $6.4 \times 10^{-3}$ & $\mathrm{~m}^{3} / \mathrm{s}$ \\
$h^{\text {min }}$ & 40 confined & $m$ \\
$h^{\text {max }}$ & 60 confined & $m$ \\
$h^{\text {min }}$ & 10 unconfined & $m$ \\
$h^{\text {max }}$ & 30 unconfined & $m$ \\
\hline
\end{tabular}

\subsection{Optimization Problem Formulation}

In this section we describe how we packaged the problem for the optimization algorithm. The objective function $f^{T}$ is discontinuous, and some of the constraints (13) and (15) are simple bounds on the variables. Implicit fi ltering, the optimization method we use in this paper, is designed to handle diffi cult objective functions and bound constraints.

If we set $n=5$, then the constraints (12) and (13) require the pumping rates to be exactly $Q_{T}^{\min } / 5$. Thus, in this situation, we need only optimize well locations and apply constraint (14). If we set $n>5$, then we must also optimize pumping rates and, therefore, all the constraints must be enforced by the optimizer. Constraint (14) is highly nonlinear while constraint (12) is not a box constraint, and neither constraint can be handled directly by the projected quasi-Newton algorithm. In this case the objective function returns a failure 
when either (12) or (14) are violated. Our implementation of implicit fi ltering will assign an artifi cial (see $\S 6.2$ ) value to the function when it returns a failure. This is a standard approach for handling nonlinear constraints in many sampling methods, [42, 9, 27].

We will fi $\mathrm{x}$ the number of wells and consider the vector of design variables

$$
Z=\left(x_{1}, \ldots, x_{n}, y_{1}, \ldots, y_{n}, Q_{1}, \ldots, Q_{n}\right)^{T} \in R^{3 n} .
$$

We defi ne the feasible set for the bound constraints as

$$
\mathcal{D}_{0}=\{Z \mid \text { (13) and (15) hold. }\}=\left\{Z \mid Z_{i}^{\min } \leq Z_{i} \leq Z_{i}^{\max }\right\} .
$$

Our optimization problem is

$$
\min _{Z \in \mathcal{D}_{0}} f^{T}(Z)
$$

where $f^{T}$ is given by (11) if (12) and (14) are satisfi ed and a failure is reported if either of (12) or (14) are violated.

\section{Implicit Filtering}

The objective function is highly nonlinear and non-convex, discontinuous because of the jumps as wells are added and deleted, and noisy, because of internal iterations in the simulators. For these reasons, as we said in $\S 1$, a conventional gradient-based optimization method may fail. A sampling method, which only evaluates the objecive function and constraints to guide the optimization, is most appropriate for this kind of problem.

In this paper we use IFFCO [10], a FORTRAN implementation of the implicit fi ltering algorithm [26, 21, 20]. We based this decision on our own familiarity with the optimizer and our past success with it on other problems of a similar mathematical nature [4, 42, 9], although we are not aware of any use of implicit fi ltering for the type of application problem of concern in this work. This choice signifi cantly influenced the decisions on handling constraints and the locations of the wells.

Implicit fi ltering has been described in detail and analyzed elsewhere. We refer the reader to [26] for the details of the algorithm and to [26, 21, 11] for convergence analysis. In $\S 6.1$ we sketch the algorithm and its implementation in IFFCO only in enough detail to explain how this choice affected the formulation of the problem. 


\subsection{THE ALGORITHM}

Implicit filtering is a projected quasi-Newton method that uses fi nite difference gradients. The difference increment is reduced as the optimization progresses, thereby avoiding some local minima, discontinuities, or nonsmooth regions that would trap a conventional gradient-based method. The problems considered in this paper are exactly the kind that the method was designed to solve.

Implicit filtering begins by rescaling the variables so that the feasible region is

$$
\mathcal{D}=\left\{\xi \mid 0 \leq \xi_{i} \leq 1\right\} .
$$

We will discuss the algorithm in terms of the scaled feasible region in (18) but the application in terms of the actual bounds (16).

To make the transition from $f^{T}$ to the scaled form, we defi ne $\xi$ componentwise by

$$
\xi_{i}=\left(Z_{i}-Z_{i}^{\text {min }}\right) /\left(Z_{i}^{\text {max }}-Z_{i}^{\text {min }}\right)
$$

and let

$$
f(\xi)=f^{T}(Z) .
$$

The optimization problem for $f$ is now

$$
\min _{\xi \in \mathcal{D}} f(\xi) \text {. }
$$

For a given difference increment (called a scale) $\delta \in(0,1 / 2]$ and $\xi \in \mathcal{D}$, we let $\nabla_{\delta} f(\xi)$ be the difference gradient whose components are

- the central difference gradient in the $i$ th coordinate direction if both of $\xi \pm \delta e_{i} \in \mathcal{D}$, or

- the one-sided difference gradient in the $i$ coordinate direction if only one of $\xi \pm \delta e_{i} \in \mathcal{D}$.

Since $\delta \leq 1 / 2$, at least one of $\xi \pm \delta e_{i} \in \mathcal{D}$. We let the stencil $S(\xi)$ be those points in the centered difference stencil that are in $\mathcal{D}$ and used in the computation of $\nabla_{\delta} f$. If

$$
f(\xi) \leq \min _{\eta \in S(\xi)} f(\eta)
$$

we say that stencil failure has occurred and terminate the quasi-Newton iteration at that scale.

If $H$ is a model Hessian, a projected quasi-Newton iteration from $\xi$ has the general form

$$
\xi(\lambda)=\mathcal{P}\left(\xi-\lambda H^{-1} \nabla_{\delta} f(\xi)\right)
$$


where $\mathcal{P}$ is the projection onto $\mathcal{D}$

$$
\mathcal{P}(\xi)_{i}= \begin{cases}0 & \text { if } \xi_{i} \leq 0 \\ \xi_{i} & \text { if } 0<\xi_{i}<1 \\ 1 & \text { if } \xi_{i} \geq 1\end{cases}
$$

In IFFCO, the step length $\lambda$ is computed with a quadratic model [10] and a step is accepted if the suffi cient decrease condition

$$
f(\xi(\lambda))-f(\xi) \leq \alpha \nabla_{\delta} f(\xi)^{T}(\xi(\lambda)-\xi),
$$

holds. In IFFCO, as is standard, $\alpha=10^{-4}$. We say that the quasi-Newton iteration is successful if

$$
\|\xi-\xi(1)\| \leq \tau \delta .
$$

The algorithmic parameter $\tau$ can have a signifi cant effect on the performance of the optimization. For the problems we consider here, however, we were able to successfully use the default value of $\tau=1$.

The fi nite difference projected quasi-Newton loop in IFFCO is summarized in algorithm fdquasi. fdquasi is a naturally parallel algorithm; all the function evaluations needed to compute $\nabla_{\delta} f$ can be done in parallel. We exploited this simple parallelism to perform the computations reported in this paper.

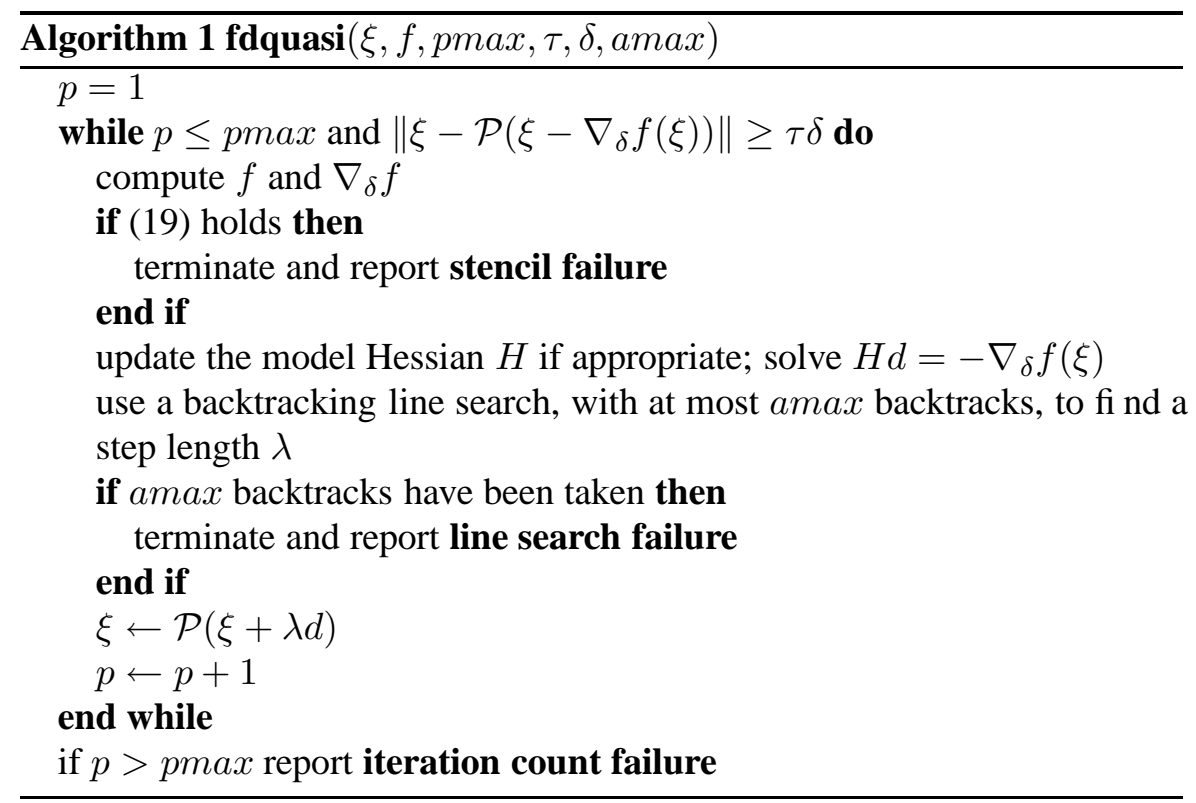

Implicit fi ltering calls fdquasi repeatedly with a sequence of scales $\left\{\delta_{k}\right\}$. Algorithm imfilter is a simple sketch. 


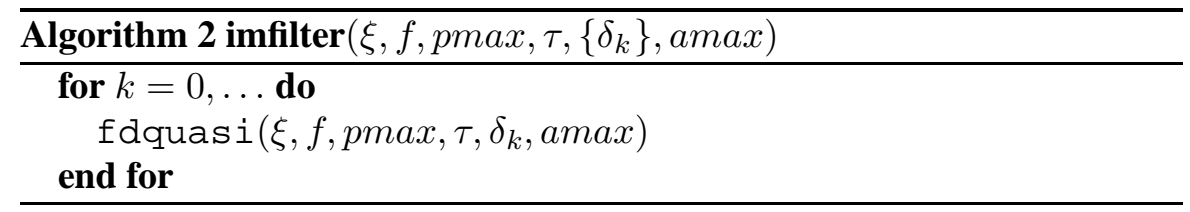

The algorithmic parameters that are important to implicit fi ltering are the limit amax on the number of step size reductions, pmax on the number of nonlinear iterations, and the parameter $\tau$ in the termination criterion. For the calculations reported here, we set $\operatorname{pmax}=100$ (the default), $\tau=1$ (the default), and $\operatorname{amax}=3$ (the default). The parameters in imfilter that control the quasi-Newton loop are the sequence of scales $\left\{\delta_{k}\right\}$. Our choice in this work was

$$
\delta_{k}=2^{-k-1}, 0 \leq k \leq 10 .
$$

The analysis of implicit fi ltering begins with the paradigm

$$
f=f_{S}+\phi
$$

where $f_{S}$ is a smooth function and $\phi$ represents the "noise" in the problem. For the theoretical convergence results in $[42,26,11]$ we assume that $\phi$ is an everywhere-defi ned function on $\Omega$ and set

$$
\|\phi\|_{S(\xi)}=\max _{\eta \in S(\xi)}|\phi(\xi)| .
$$

One can show that if either (19) or (21) hold, that

$$
\left\|\mathcal{P}\left(\xi-\nabla f_{S}(\xi)\right)\right\|=O\left(\delta+\|\phi\|_{S(\xi)} / \delta\right) .
$$

The convergence theory for implicit fi ltering [26, 21, 11] are based on (23).

IFFCO supports the SR1 [7, 18] and the BFGS [41, 8, 19, 22] quasiNewton models of the Hessian. We used the SR1 update in this paper. In our experience the SR1 update performs better for bound-constrained problems.

Implicit fi ltering can be restarted after it terminates and the convergence theory [21] is stronger if one does that. In practice, restarting usually has no effect. For the problems in this paper, however, we had to restart IFFCO once to obtain consistently good results.

\subsection{FAILURE OF THE FUNCTION}

IFFCO responds to a failure of $f$ in two ways. If the failed function evaluation $f^{T}(z)$ is part of the evaluation of $\nabla_{h} f^{T}(\xi)$, then an artifi cial value [9] of

$$
f^{*}+10^{-6}\left|f^{*}\right|
$$


is assigned to $f(z)$. Here $f^{*}$ is the largest function value in the stencil $S(\xi)$. If the function evaluation failure is part of the line search, the the value $f$ scale is assigned to $f^{T}$.

fscale is an approximation to the maximum value of $f^{T}$ in the feasible set $\mathcal{D}_{0}$ for the bound constraints (16). We set f scale to $20 \%$ more than the value of $f$ at the initial iterate in this paper.

This approach to handling constraints is natural if the failure of the objective function is a consequence of, for example, an internal iteration's failure to converge. In the case of the problem considered here, while the constraints are directly specifi ed by (14), the evaluation of $h_{i}$ requires a call to the simulator which, as a function of the well locations, is highly nonlinear even for the continuous problem. For the discrete problem considered here, where the well locations are rounded to grid points before the call to the simulator, the constraint function is discontinuous.

\section{Evaluation of the Objective Function}

IFFCO requires an external subroutine to evaluate the objective function $f^{T}$. To do this we must compute the hydraulic head values, $\left\{h_{i}\right\}$, at the well locations $\left\{\left(x_{i}, y_{i}\right)\right\}$ for a given set of pumping rates $\left\{Q_{i}\right\}$. Computation of $\left\{h_{i}\right\}$ uses a groundwater fbw simulator to solve (1). For this work we use the U.S. Geological Survey code MODFLOW-96 [31]. MODFLOW is a blockcentered fi nite difference code that simulates saturated groundwater fbw and allows for a variety of boundary conditions and irregular physical domains. MODFLOW is widely used and well supported.

A MODFLOW simulation requires an input fi le containing the location and pumping rates of the wells in the model. If $n>5$, each function evaluation requires a new set of pumping rates and thus the MODFLOW well fi le must be created each time the objective function is evaluated. Moreover, once the MODFLOW simulation is complete, the values of $h_{i}$ must be extracted from the MODFLOW output fi le. A typical function evaluation is shown in Figure 2.

To generate the necessary data fi les to run MODFLOW we used the Groundwater Modeling System (GMS), version 3.1. GMS is a modular interface to a variety of fbw and transport codes, including MODFLOW. GMS has a graphical

environment that allows the user to generate grids, defi ne characteristics of the porous media, and visualize solutions. GMS was used to generate the starting heads for (7), to create the necessary data fi les for MODFLOW, to determine an appropriate initial iterate for the optimization, and then again to test the results of the optimizer. 


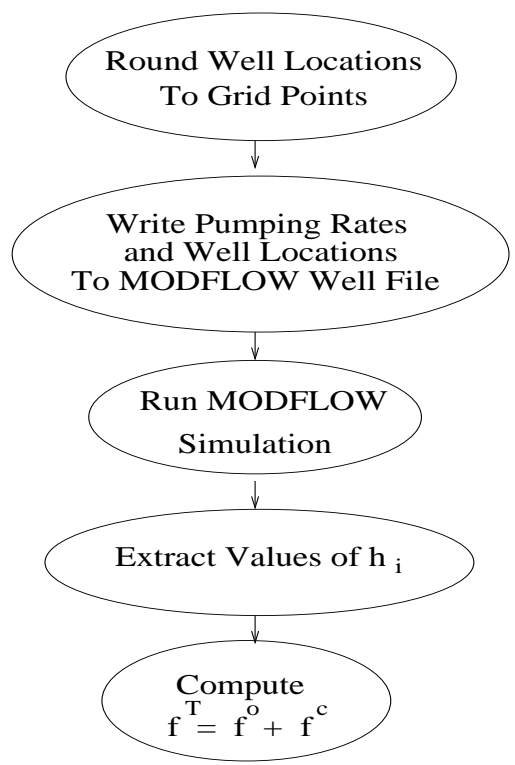

Figure 2. Objective Function Evaluation

\section{Numerical Results}

We consider two formulations of the design space. For this work, the installation cost of an extraction well, which is roughly $\$ 20,000$, is high compared to the annual operating cost which is roughly $\$ 1,000$. Since $n=5$ wells extracting at $Q^{\text {emax }}=-0.0064\left(\mathrm{~m}^{3} / \mathrm{s}\right)$ satisfi es the water supply demand (12) exactly, one obvious formulation is to fi $\mathrm{x} n=5$ and $\left\{Q_{i}\right\}_{i=1}^{5}=-0.0064$ and seek the optimal locations, $\left\{\left(x_{i}, y_{i}\right)\right\}_{i=1}^{5}$ to minimize only the operational $\operatorname{cost}\left(f^{o}\right.$ in (11)). We also include a formulation in which we start with $N=6$ wells and seek the optimal locations and pumping rates to minimize (11), $f^{T}=f^{c}+f^{o}$. Intuitively, since the installation cost is so high, we would expect the fi ve well confi guration to have the lowest cost.

We examined the performance of one gradient-based code, the FDNIPS solver from the OPT++ v2.0 [33] framework. This code is a nonlinear interior point code based on the work in [17, 2, 3]. The code uses fi nite difference gradients, either trust region or line search globalization, and a choice of three merit functions. We tried several combinations of the options. In every case the optimization failed after 1000 calls to the function or failed because the line search had reduced the step length 40 times without a suffi cient decrease in the merit function.

For comparison, we include results obtained with a simple genetic algorithm (GA). The performance of a GA for constrained optimization problems often depends strongly on a number of factors including the method used to encode the design variables, the choice of selection, crossover, and mu- 
tation operators, and the manner in which constraints are handled [36, 29]. Here, we consider a single-objective GA which incorporates both real- and binary-coded variables, and uses binary tournament selection [15]. For the real-coded variables, the simulated binary crossover (SBX) operator $[15,14]$ with polynomial mutation is used while single-point crossover with bitwise mutation are used for binary-coded variables.

An approach based on [12] is used to include constraints without the use of penalty parameters. Box constraints such as those in (13) are enforced automatically in the generation of candidate design variables, while a constraint such as (12) is formulated as a non-negative function $g(Z) \geq 0$. The GA tournament selection process is then modifi ed to account for the three scenarios: (1) when two feasible solutions are compared, the one with lower objective value is preferred; (2) when a feasible and infeasible solution are compared, the feasible one is taken; and (3) when two infeasible solutions are compared the one with lower overall constraint violation is preferred [12].

Parameters like the population size, number of generations, as well as the probabilities and distribution indexes chosen for the crossover and mutation operators effect the performance of a GA [36, 29]. For the purposes of our comparison, we wished to limit the number of simulations performed by the GA to a range of 2 to 3 times the number required by IFFCO. Since the total number of objective function evaluations is roughly the product of the population size and number of generations, this restricted our choices to fairly small populations and few generations. We also wished to use similar parameter values across the various problems. Although we did not perform a systematic study to find the best possible combinations, we experimented with a series of population sizes, numbers of generations, and crossover and mutation parameters to fi nd a combination that gave representative performance for each of the problems we considered. Unless noted, the values used are listed Table III.

The GA code used is implemented in C and is available for download from [13]. The user is required to implement problem-specifi c routines for evaluating objective functions and constraints. 
Table III. GA parameters

\begin{tabular}{ll}
\hline 30 & size of population \\
30 & number of generations \\
0.9 & crossover probability \\
0.1 & real-coded mutation probability \\
20 & distribution index for real-coded crossover \\
10 & distribution index for real-coded mutation \\
0.5 & binary-coded mutation probability \\
0.1 & niching-parameter for constraints
\end{tabular}

\subsection{Spatial Discretization}

We use the same spatial discretization for both formulations. For the confi ned aquifer we discretize the domain $\Omega=[0,1000] \times[0,1000] \times[0,30](\mathrm{m})$ on an equally spaced $50 \times 50 \times 10$ grid. For the unconfi ned aquifer, we used MODFLOW to determine the saturated domain $\Omega_{u n c}=[0,1000] \times[0,1000] \times$ $[0,27] \subset \Omega$ and then discretized $\Omega_{u n c}$ on an equally spaced $50 \times 50 \times 10$ grid.

\subsection{FiVE WELL FORMULATION}

\subsubsection{Initial Iterate}

IFFCO requires a feasible initial iterate. Figure 3 shows the steady state fbw fi eld for the confi ned aquifer. Since the head value is high in the lower left corner we initially placed one well there. After the wells are activated, the constraint on the drawdown is violated if the wells are too close together. We looked at several different initial iterates until we found one that satisfi ed the drawdown constraint for both the confi ned and unconfi ned aquifer. We found that placing the remaining four wells close to the specifi ed head boundaries and signifi cantly apart from each other was feasible for both physical domains. Figure 4 shows the relative location of the wells and the pressure head fi eld for the confi ned aquifer with the wells pumping at the initial iterate. Note the same initial well locations were used for both aquifers. 


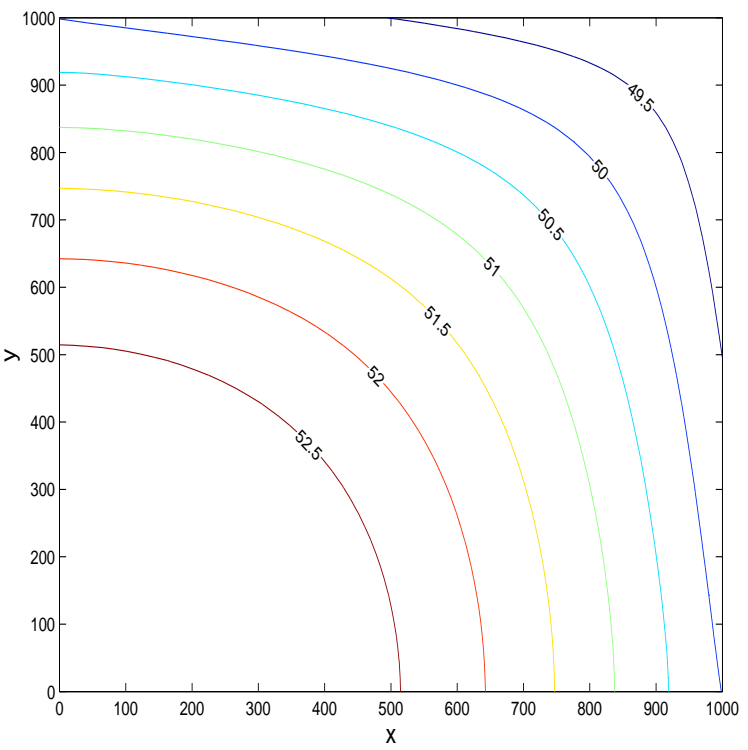

Figure 3. Steady state head, confined aquifer

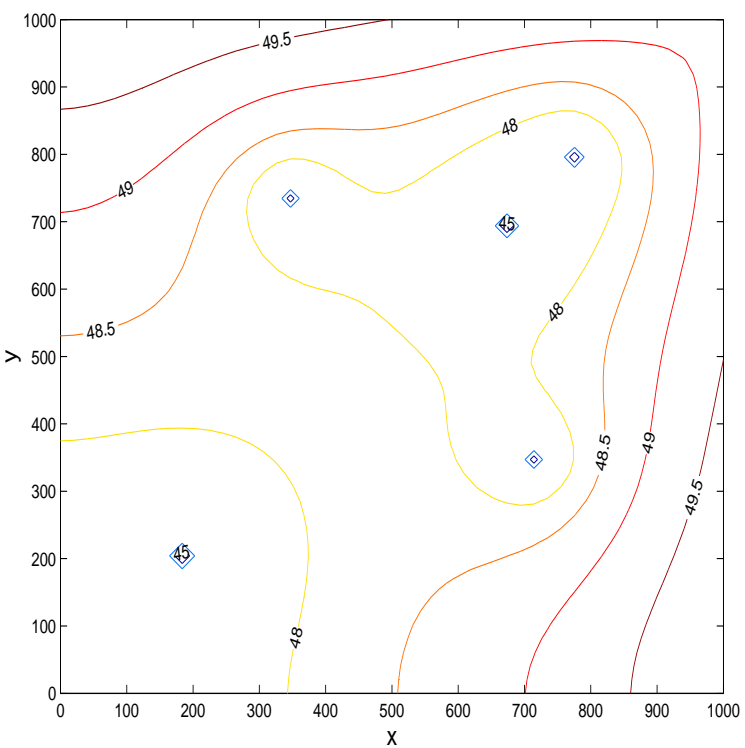

Figure 4. Initial Iterate 
We refer to the confi ned aquifer as CON and unconfi ned aquifer as UNC. The function value at the initial iterate was $\$ 23,204$ for CON and $\$ 26,958$ for UNC. Table IV shows the minimum cost found and the number of calls to MODFLOW for both optimizers and aquifers. IFFCO reduced the cost by $6 \%$ for the confi ned aquifer and $11 \%$ for the unconfi ned aquifer. For both aquifers, the minimum cost found by the GA after 10 generations was 5\% higher than the cost found by IFFCO-which is high considering the decrease from the initial iterate. Table $\mathrm{V}$ shows the initial $\mathrm{x}-\mathrm{y}$ coordinates for the 5 wells and the optimal locations for each aquifer. The well locations at the optimal point lie on the boundary constraint, (15). This is physically reasonable, since the head values are higher in that region due to the Dirichlet boundary conditions. The GA's cost was higher than IFFCO's because well 5 in the confi ned case and well 1 in the unconfi ned case are not relocated close enough to the Dirichlet boundary conditions where the head values are higher.

In our evaluation of performance, we count only the expensive calls to the simulator as opposed to cumulative calls to the function. This is the approach taken in [6]. To see how this is a more realistic way to measure cost, consider the case where the linear constraint (12) is violated. One can detect this violation, and return a failure for $f$, without calling the expensive fbw simulator. IFFCO is being modifi ed to make it easy for the user to evaluate cost at a fi ner granularity than this, to allow for the use of multiple simulators within the evaluation of the objective function and constraints. While this is a simple change in a serial code, correctly counting the calls to the various simulators in a parallel implementation requires considerable care.

Figure 5 is a plot of the value of the objective function against the cumulative number of calls to the simulator. IFFCO is currently being modifi ed to allow the user to easily count calls to the objective function and calls to the individual simulators that are used to compute it. We set a function evaluation budget of 10,000 for this work and IFFCO converged to an optimal point within approximately $3 \%$ of the budget, terminating the optimization based on the sequence of fi nite difference scales. Figure 5 shows that after only roughly 100 function evaluations, the objective function does not decrease signifi cantly.

Figures 6 and 7 show the head contours in the layers containing the wells with the wells at the optimal locations.

\subsection{SIX WELL FORMULATION}

The results above are based on the heuristic that installing the minimum number of wells (5) that meet the extraction target is the best approach. To test this, we compared the fi ve well confi guration with all wells pumping at the maximum extraction rate to a six well confi guration with both locations 
Table IV. Cost: 5 Wells

\begin{tabular}{|c|c|c|c|}
\hline Optimizer & Problem & $\min \mathrm{f}$ & MODFLOW Calls \\
\hline IFFCO & CON & $\$ 21,830$ & 275 \\
GA & CON & $\$ 22,822$ & 330 \\
IFFCO & UNC & $\$ 23,930$ & 302 \\
GA & UNC & $\$ 25,164$ & 328 \\
\hline
\end{tabular}

Table V. Optimal Locations

\begin{tabular}{|c|c|c|c|c|}
\hline $\begin{array}{c}\text { Init_Co } \\
(\mathrm{m})\end{array}$ & $\begin{array}{c}\text { IFFCO } \\
\text { CON (m) }\end{array}$ & $\begin{array}{c}\text { GA } \\
\text { CON (m) }\end{array}$ & $\begin{array}{c}\text { IFFCO } \\
\text { UNC (m) }\end{array}$ & $\begin{array}{c}\text { GA } \\
\text { UNC (m) }\end{array}$ \\
\hline $\mathrm{X}(1) 350.0$ & 401.7 & 655.1 & 464.2 & 600.0 \\
$\mathrm{Y}(1) 725.0$ & 800.0 & 737.8 & 800.0 & 216.2 \\
\hline $\mathrm{X}(2) 775.0$ & 800.0 & 794.3 & 800.0 & 397.5 \\
$\mathrm{Y}(2) 775.0$ & 800.0 & 782.3 & 800.0 & 774.4 \\
\hline $\mathrm{X}(3) 675.0$ & 776.9 & 755.6 & 800.0 & 796.5 \\
$\mathrm{Y(3)} 675.0$ & 481.1 & 203.1 & 445.4 & 706.0 \\
\hline $\mathrm{X}(4) 200.0$ & 138.2 & 569.3 & 138.2 & 149.7 \\
$\mathrm{Y(4)} \mathrm{200.0}$ & 800.0 & 798.3 & 800.0 & 771.9 \\
\hline $\mathrm{X}(5) 725.0$ & 798.4 & 303.8 & 800.0 & 799.3 \\
$\mathrm{Y(5)} 350.0$ & 168.9 & 501.6 & 144.8 & 513.3 \\
\hline
\end{tabular}

and pumping rates as decision variables. We included the installation cost $\left(f^{c}\right.$ in (11)) in the objective function for these runs. If the six well problem is initialized with all wells pumping at the maximum extraction rate, then one well is removed from the design in the course of the optimization and the minimum function value is within $0.2 \%$ of that found with the original fi ve well confi guration. If the six wells are initialized with

$$
Q_{i}=Q_{T}^{\min } / 6, i=1 \ldots 6,
$$

which is a feasible and sensible initial iterate, then a suboptimal point is found. All wells remain pumping close to the initial pumping rates, although the locations align with the specifi ed head boundary conditions.

The objective function for the six well problem contained a large installation cost, $f^{c}$, per well. A common approach for such conditions is to use 


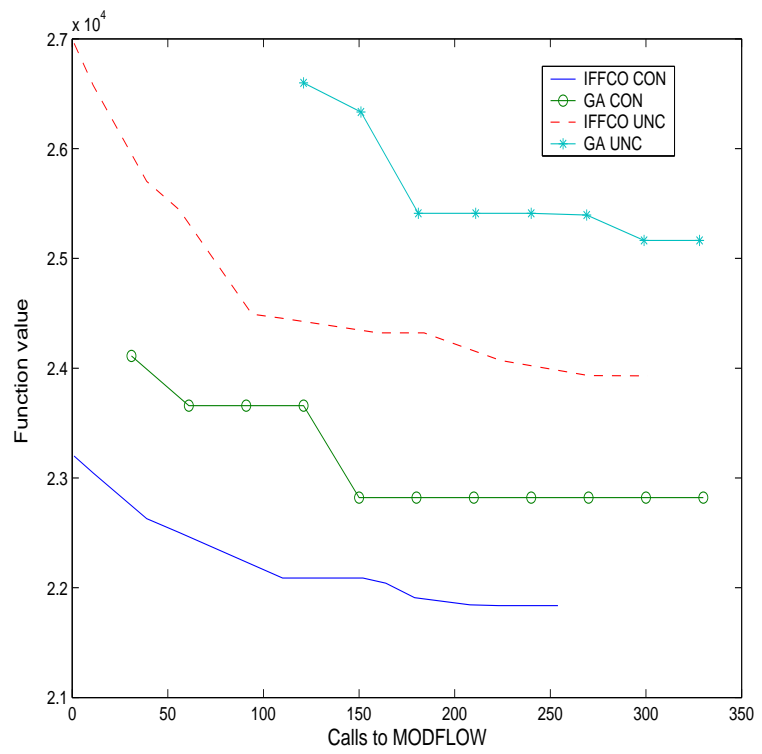

Figure 5. Decrease in Function Values

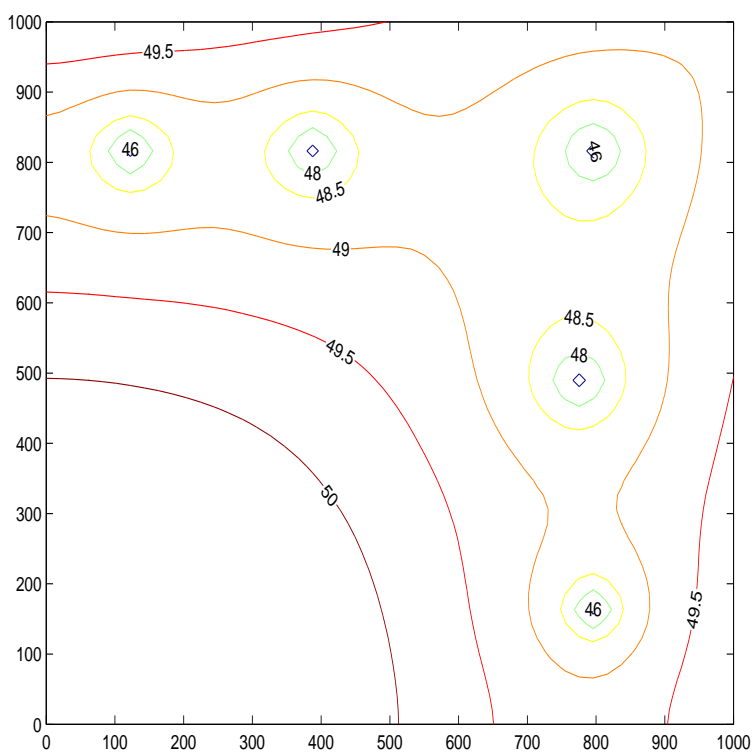

Figure 6. Confined Aquifer

a mixed-integer formulation [37, 44, 30, 35]. Specifi cally, given the fact that $f^{c}$ was signifi cantly larger than $f^{o}$ and that a minimum of fi ve wells were required to satisfy the extraction target, a reasonable way to recast the problem was to include an integer variable indicating which, if any, well should be removed from the design. It was straightforward to include an integer variable 


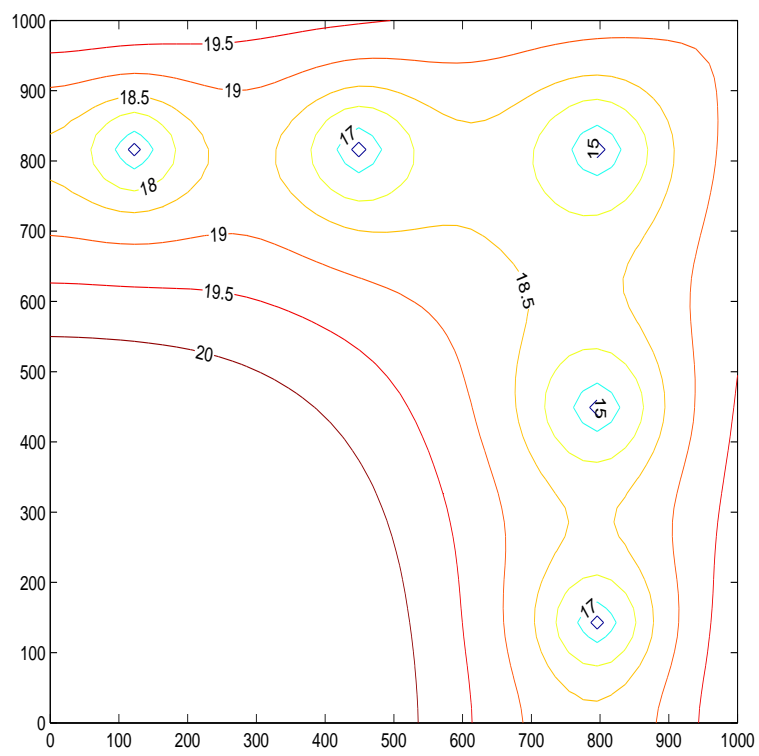

Figure 7. Unconfined Aquifer

$s$ ranging from 1 to 8 in the GA's formulation. A value of $s$ in the range $1, \ldots, 6$ resulted in shutting off the corresponding well, while $s=7,8$ led to six well designs. In addition, a well was removed from the design if its pumping rate fell below the installation threshold, regardless of the value of $s$. The unequal ranges associated with fi ve and six well designs skewed the GA's formulation to favor fi ve-well designs. This reffected our heuristic that installing the minimum number of wells was likely to be cheaper than a six-well design.

Table VI shows the minimum cost and the number of calls to MODFLOW for both optimizers, aquifers for the better initial iterate. The cost at the initial iterate was $\$ 170,972$ for the confi ned aquifer and $\$ 152,878$ for the unconfi ned aquifer. Both the GA and IFFCO were able to remove one well from the design, resulting in a solution comparable to the fi ve well confi guration and reducing the cost by roughly $20 \%$. For the suboptimal initial iterate, IFFCO was only able to decrease the cost $1 \%$. The GA did not fi nd anything better than the initial iterate in 30 generations (over 100 function evaluations) for either aquifer with the suboptimal initial iterate included in the initial population.

\subsection{OPTIMIZATION LANDSCAPES}

The fi ve well confi guration does not have a discontinuous installation cost. To get a better understanding of the objective function for this formulation, we fi xed wells 2-5 and computed the cost while letting the $x$ and $y$ coordi- 
Table VI. Cost: 6 Wells

\begin{tabular}{|c|c|c|c|}
\hline Optimizer & Problem & $\min \mathrm{f}$ & MODFLOW Calls \\
\hline IFFCO & $\mathrm{CON}$ & $\$ 140,237$ & 346 \\
GA & $\mathrm{CON}$ & $\$ 140,628$ & 464 \\
$\mathrm{IFFCO}$ & $\mathrm{UNC}$ & $\$ 124,582$ & 327 \\
GA & $\mathrm{UNC}$ & $\$ 127,069$ & 161 \\
\hline
\end{tabular}

nates for well 1 vary between 20 and 800 meters. Figure 8 shows the cost landscape near the initial iterate for the confi ned aquifer and Figure 9 shows the landscape near the initial iterate for the unconfi ned aquifer. The peaks in the landscapes occur when two wells get close together, making the head values low and hence the operational cost higher. When two wells get too close they violate (14), leaving a small infeasible region inside each of the peaks. These peaks also make the landscapes nonconvex and introduce local minima. When we try to evaluate the function at an infeasible point, we do not plot an artifi cial value. Note that only a subset of $\Omega$ is feasible, especially for the unconfi ned aquifer near the initial iterate. The high infeasibility was due to repeated violation of the head constraint (14), which is why the unconfi ned case is more challenging. There are also small discontinuities apparent in the landscapes since we round real numbers to grid locations to run the fbw simulator.

Figures 10 and 11 are the surfaces obtained when wells 2-5 are set at the optimal locations for the confi ned and unconfi ned aquifers. 


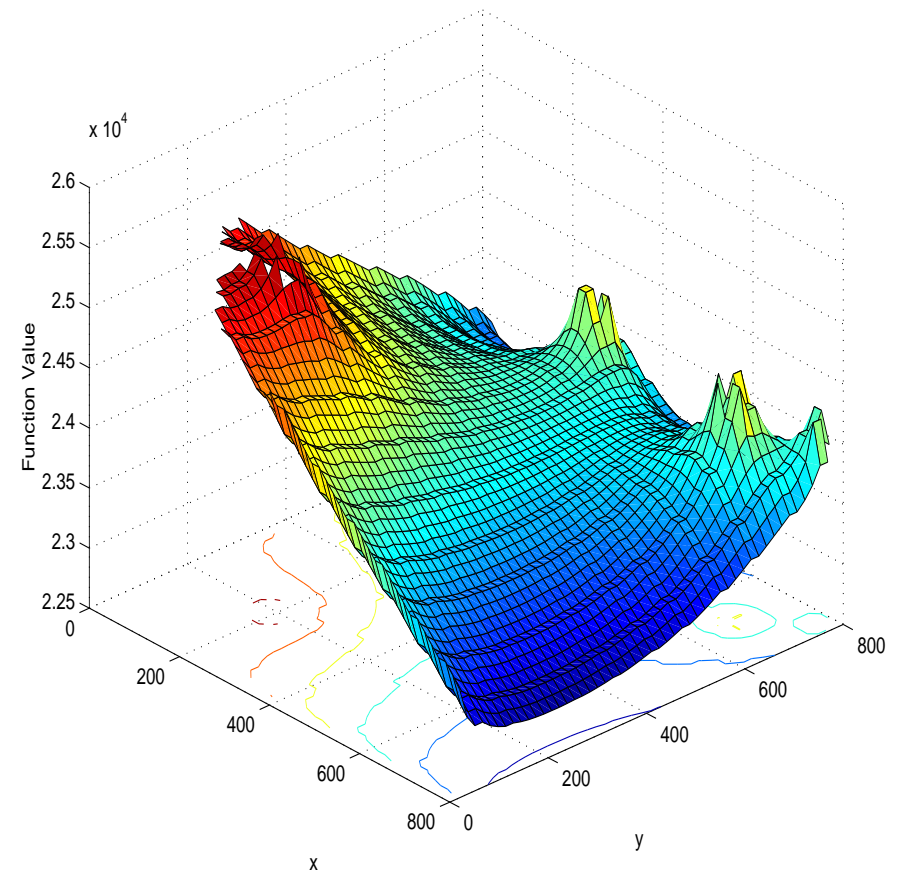

Figure 8. Landscape near initial iterate: confined aquifer

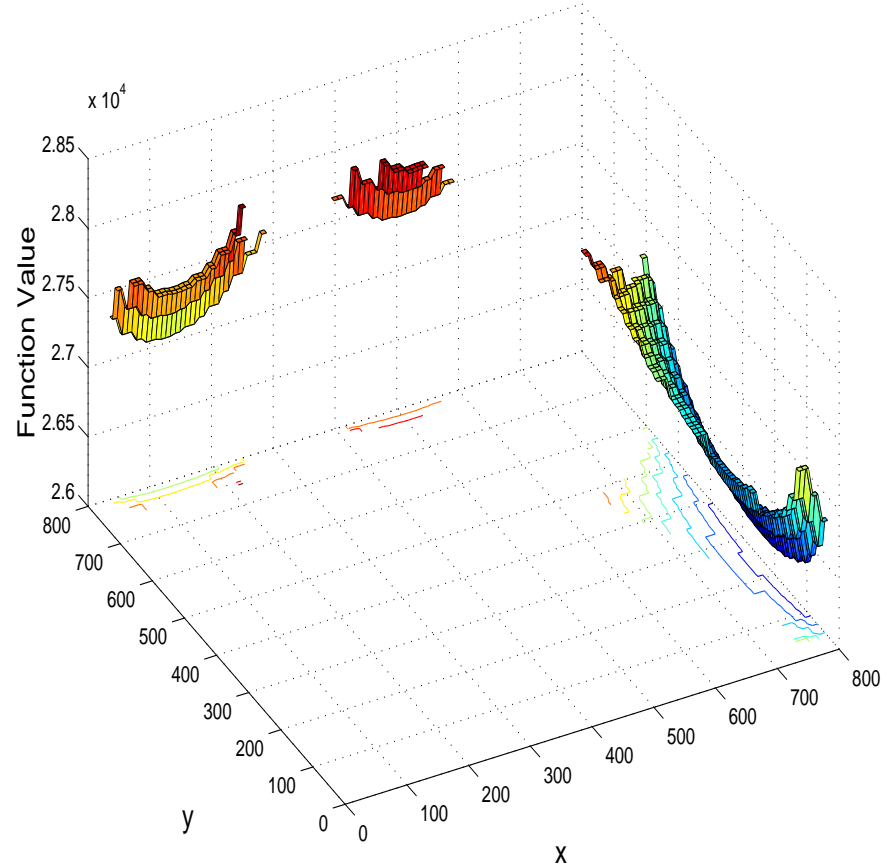

Figure 9. Landscape near initial iterate: unconfined aquifer 


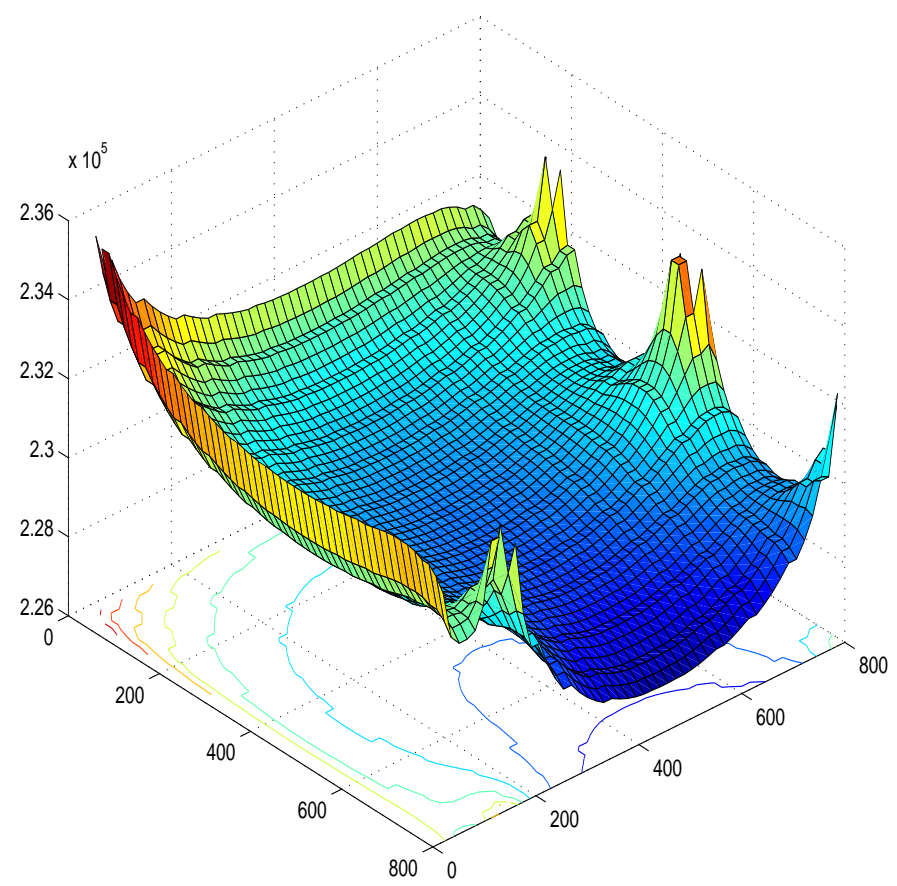

Figure 10. Landscape near solution: confined aquifer

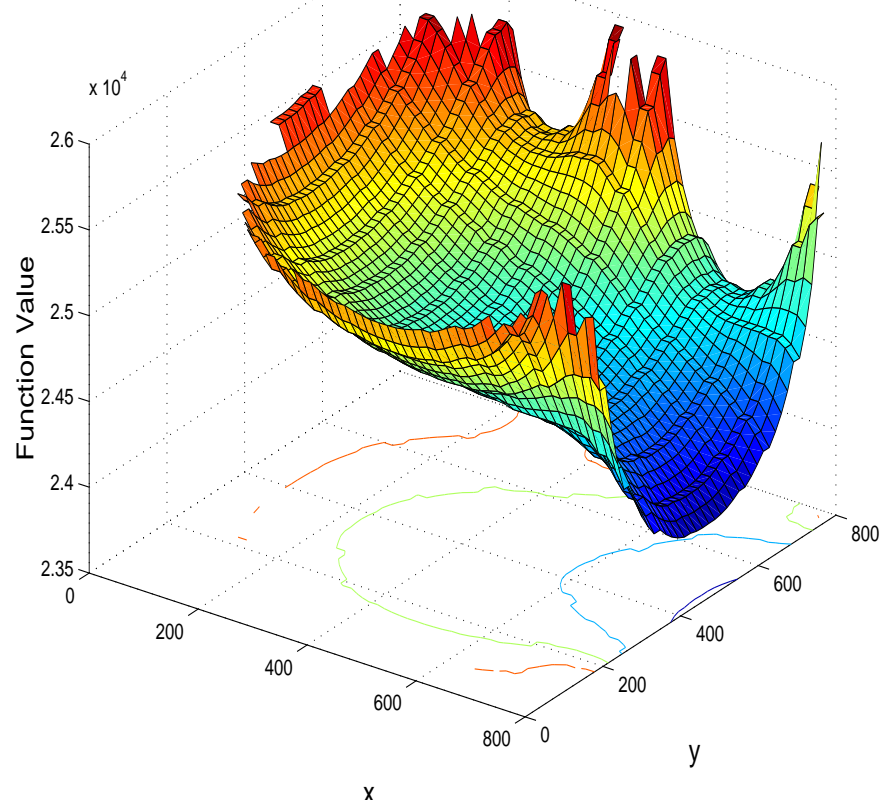

Figure 11. Landscape near solution: unconfined aquifer 


\subsection{DISCUSSION}

- The numerical results indicate that the six well formulation is much more diffi cult than the fi ve well formulation since one well must be removed from the design while the remaining wells extract at the maximum extraction rate. IFFCO did well on the the six well formulation with an initial iterate with all wells extracting at the maximum rate. In the other case a local minimum is found. With or without the mixed-integer formulation, the six well problem proved diffi cult for the GA, since removing a well only led to a feasible iterate if the other fi ve were pumping at the maximum rate. The GA was able to fi nd a feasible fi ve well solution when the better initial iterate was included as a member of the initial population. With a completely random initial population or with the sub-optimal initial iterate included, the GA solution for the confi ned aquifer problem was a sub-optimal six well design. The same was true for the unconfi ned case. However, the GA with a completely random population was unable to find a feasible iterate for the unconfi ned problem even when using a population size of two hundred and running for two hundred generations.

- We also considered the possibility that, over a longer time period, the six well model with the suboptimal initial iterate may be superior to the fi ve well model. We ran both the confi ned and unconfi ned problems for one year to determine the annual operational cost. One can see that a time of roughly 130 years for the confi ned aquifer and 90 years for the unconfi ned aquifer is needed to obtain a lower using the six well model. Hence the fi ve well results are the most realistic. We ran both problems again with the longer time horizons to confi rm that the six well model would outperform the fi ve well model.

- It is common in practice to start with a large, fi xed grid of wells and seek only the optimal pumping rates, removing wells from the design as needed. We tried this approach, using $N=16$ so that we seek the optimal rates, $\left\{Q_{i}\right\}_{i=1}^{16}$ to minimize $f^{T}=f^{c}+f^{o}$. Neither IFFCO nor the GA was able to converge to the fi ve well solution. We tried several different initial iterates for IFFCO and at best, in 429 function evaluations, IFFCO had left 10 wells in the design and reduced the cost from $\$ 424,123$ to $\$ 262,558$ for the confi ned aquifer. The results were similar for the unconfi ned aquifer, but it was even more challenging to fi nd a feasible initial iterate. We performed several experiments with IFFCO and with a good initial iterate, IFFCO was able to converge to the fi ve well solution with up to $N=9$ candidate wells initially fi xed on the grid. With a relatively large number of fi xed wells, it was again natural to use a mixed-integer formulation for the GA. This time, the design variables consisted of a rate and binary "on-off" switch for each of the

$N=16$ wells. Starting from a random initial population, the GA produced a six well design for both the confi ned and unconfi ned problems. The fi nal cost 
for the confi ned aquifer design was $f^{T}=\$ 161,588$ after 510 function evaluations, while the unconfi ned aquifer solution had a cost of $f^{T}=\$ 142,755$ after 359 function evaluations.

- This application is challenging for formulations with $N>5$ wells since wells must be removed from the design space to decrease the installation cost. Removing wells from the design is an active area of research and numerous approaches exist for approximating fi xed costs with continuous functions (see [32] and the references therein). Our approach for removing a well if $\left|Q_{i}\right|<10^{-4}$ results in a discontinuous fi xed cost. This approach was implemented due to its simplicity, but also because the implicit fi ltering algorithm is designed for problems with discontinuous landscapes. As the number of wells increased, it was more diffi cult to fi nd a feasible initial iterate and even for the six well formulation, we found that IFFCO required a decent initial iterate. A possible remedy for this might be to use another optimization routine as an initial iterate generator for IFFCO. This will be the subject of future work. 


\section{Conclusions}

This work was an initial analysis of a subset of the community problems proposed in [30]. The formulation of the objective function and constraints are discontinuous and have local minima as the optimization landscapes verify. These features are the reason for the failure of the gradient-based method (see $\S 8$ ). As pointed out in [30], deterministic sampling methods have not been used to their full potential in the subsurface optimization community. In this work we found a solution to the well-fi eld design application with the implicit filtering algorithm and we compared our results to those obtained with a simple genetic algorithm.

We found these problems to be challenging for several reasons. A minimal cost is obtained with few wells pumping at large rates. For these problems, local minima exist when more wells are extracting at low costs. A large decrease in the objective function occurs when a well is removed from the design space. The installation cost for this work is discontinuous, yet we

found that with good initial data, that the implicit fi ltering algorithm could perform well despite the discontinuous formulation for $N<9$. Another challenge is that the feasible region, especially for the unconfi ned aquifer, is small. Although implicit fi ltering started with a feasible initial iterate, much experimental work was done to fi nd one. The genetic algorithm was unable to find a feasible point for the six well formulation and required a feasible point in the initial population in order for the optimization to progress for the unconfi ned aquifer.

We can extend this study to improve the solution for this type of problem. - IFFCO requires a feasible initial iterate, and the numerical results show that a good initial iterate is needed for the optimization. Surrogate models based on statistical sampling $[5,6]$ may be a good way to explore design space for good initial iterates.

- As pointed out in [30], a more accurate realization of the subsurface is needed for solutions of this class of problems to be used in decision making. Adding heterogeneities to the domain would create a more realistic snapshot of the subsurface yet would make the optimization landscapes much more challenging. More robust optimization techniques may be needed as the conceptual domain becomes more realistic.

- We used MODFLOW to simulate fbw for a well-fi eld design application, despite the simulator's simple well model. The real-valued well location that is output from the optimizer is rounded to a grid location. For a more accurate solution, a simulator that is able to more accurately resolve fbw around the well is essential. A well model that need not place wells at the center of a cell would be ideal.

- This was the fi rst attempt at obtaining a solution to any of the problems proposed in [30]. An in-depth comparison of sampling methods, including those 
that use a surrogate response surface, is currently being done and is necessary before any solid conclusions can be made on which method performs best for this class of problems.

\section{Downloading and Running the Test Problems}

The problems can be obtained from

http: //www 4 .ncsu.edu/ ctk/community.html

The test problems are packaged as compressed UNIX tar files. The serial codes are for the g77 compiler and have been tested on SUN SparcStations running Solaris, various Intel platforms running Red Hat Linux 7.3 and 8.0, and an Apple Macintosh G4 running OSX 10.2. The MPI version of the codes has been tested on an IBM-SP3 and a DELL Linux server. IFFCO is included in the packages. The README fi les in the main directory explain how to assemble the fi les and interpret the results.

MODFLOW can be obtained directly from the USGS at the URL

http://water.usgs.gov/software/modflow-96.html

The USGS provides compiled executables for SUN, SGI, and DOS systems, as well as UNIX source. Our packages provide makefi les for some other UNIX environments.

\section{Acknowledgments}

The research of KRK, CEK, CTK, RWD, and JPR was supported in part by National Science Foundation grants DMS-0070641, DMS-0112542, Army Research Offi ce grants DAAD19-02-1-0391 and DAAD19-02-1-0111, and a Department of Education GAANN fellowship. The research of CTM and MWF was partially supported by National Institute of Environmental Health Sciences grant P42 ES05948-02 and National Science Foundation grant DMS0112653. Partial support of this work was also provided by the National Science Foundation through DMS-0112069 to the Statistical and Applied Mathematical Sciences Institute in Research Triangle Park, where a portion of this work was done. Some of the computational support was provided by the North Carolina Supercomputing Center.

The authors thank John Dennis, Alex Mayer, and Patrick Reed for several useful discussions. 


\section{References}

1. Aly, A. H. and R. C. Peralta: 1999 , 'Comparison of a genetic algorithm and mathematical programming to the design of groundwater cleanup systems'. Water Resources Research 35(8), 2415-2425.

2. Argaez, M. and R. A. Tapia: 2001, 'On the Global Convergence of a Modified Augmented Lagrangian Linesearch Interior-Point Newton Method for Nonlinear Programming'. J. Optim. Theory Appl. 114, 1-25.

3. Argaez, M., R. A. Tapia, and L. Velazquez: 2002, 'Numerical Comparisons of PathFollowing Strategies for a Primal-Dual Interior-Point Method for Nonlinear Programming'. J. Optim. Theory Appl. 114, 255-272.

4. Battermann, A., J. M. Gablonsky, A. Patrick, C. T. Kelley, T. Coffey, K. Kavanagh, and C. T. Miller: 2002, 'Solution of A Groundwater Control Problem with Implicit Filtering'. Optimization and Engineering 3, 189-199.

5. Booker, A. J.: 1994, 'DOE for computer output'. Technical Report BCSTECH-94-052, Boeing Computer Services, Seattle, WA.

6. Booker, A. J., J. E. Dennis, P. D. Frank, D. B. Serafini, V. Torczon, and M. W. Trosset: 1999, 'A rigorous framework for optimization of expensive functions by surrogates'. Structural Optimization 17, 1-13.

7. Broyden, C. G.: 1967, 'Quasi-Newton methods and their application to function minimization'. Math. Comp. 21, 368-381.

8. Broyden, C. G.: 1969, 'A new double-rank minimization algorithm'. AMS Notices 16, 670.

9. Carter, R., J. M. Gablonsky, A. Patrick, C. T. Kelley, and O. J. Eslinger: 2001, 'Algorithms for Noisy Problems in Gas Transmission Pipeline Optimization'. Optimization and Engineering 2, 139-157.

10. Choi, T., O. Eslinger, P. Gilmore, C. Kelley, and H. Patrick: 2001, 'User's guide to IFFCO'.

11. Choi, T. D. and C. T. Kelley: 2000, 'Superlinear Convergence and Implicit Filtering'. SIAM J. Optim. 10, 1149-1162.

12. Deb, K.: 2000, 'An efficient constraint handling method for genetic algorithms'. Computer Methods in Applied Mechanics and Engineering 186(2-4), 311-338.

13. Deb, K.: 2003, 'KanGal Homepage'. Indian Institute of Technology Kanpur http://www.iitk.ac.in/kangal.

14. Deb, K. and H. Beyer: 2001, 'Self-Adaptive Genetic Algorithms with Simulated Binary Crossover'. Evolutionary Computation Journal 9(2), 197-221.

15. Deb, K. and A. R.B.: 1995, 'Simulated binary crossover for continuous search space'. Complex Systems 9, 115-148.

16. Dennis, J. E. and V. Torczon: 1991, 'Direct Search Methods on Parallel Machines'. SIAM J. Optim. 1, $448-474$.

17. El-Bakry, A. S., R. A. Tapia, T. Tsuchiya, and Y. Zhang: 1996, 'On the formulation and theory of the Newton interior-point method for nonlinear programming'. J. Optim. Theory Appl. 89, 507-541.

18. Fiacco, A. V. and G. P. McCormick: 1990, Nonlinear Programming, No. 4 in Classics in Applied Mathematics. Philadelphia: SIAM.

19. Fletcher, R.: 1970, 'A new approach to variable metric methods'. Comput. J. 13, 317322.

20. Gilmore, P.: 1993, 'An Algorithm for Optimizing Functions with Multiple Minima'. Ph.D. thesis, North Carolina State University, Raleigh, North Carolina.

21. Gilmore, P. and C. T. Kelley: 1995, 'An implicit filtering algorithm for optimization of functions with many local minima'. SIAM J. Optim. 5, 269-285. 
22. Goldfarb, D.: 1970, 'A family of variable metric methods derived by variational means'. Math. Comp. 24, 23-26.

23. Hooke, R. and T. A. Jeeves: 1961, 'Direct search solution of numerical and statistical problems'. Journal of the Association for Computing Machinery 8, 212-229.

24. Huang, C. and A. S. Mayer: 1997, 'Pump-and-treat optimization using well locations and pumping rates as decision variables'. Water Resources Research 33(5), 1001-1012.

25. Jones, D. R., C. C. Perttunen, and B. E. Stuckman: 1993, 'Lipschitzian Optimization Without the Lipschitz Constant'. J. Optim. Theory Appl. 79, 157-181.

26. Kelley, C. T.: 1999, Iterative Methods for Optimization, Vol. 16 of Frontiers in Applied Mathematics. Philadelphia, PA: SIAM, first edition.

27. Lewis, R. M. and V. Torczon: 2000, 'Pattern Search Algorithms for Linearly Constrained Minimization'. SIAM J. Optim. 10, 917-941.

28. Marryott, R. A., D. E. Dougherty, and R. L. Stollar: 1993, 'Optimal Groundwater Management .2. Application of Simulated Annealing to a Field-Scale Contamination Site'. Water Resources Research 29(4), 847-860.

29. Mayer, A., C. Kelley, and C. Miller: 2002a, 'Optimal design for problems involving flow and transport phenonmena in saturated subsurface systems'. Advances in Water Resources 12, 1233-1256.

30. Mayer, A. S., C. T. Kelley, and C. T. Miller: 2002b, 'Optimal Design for Problems Involving Flow and Transport Phenomena in Saturated Subsurface Systems'. Advances in Water Resources 12, 1233-1256.

31. McDonald, M. and A. Harbaugh: 1988, 'A Modular Three Dimensional Finite Difference Groundwater Flow Model'. U.S. Geological Survey Techniques of Water Resources Investigations.

32. McKinney, D. C. and M.-D. Lin: 1995, 'Approximate Mixed-Integer Nonlinear Programming Methods for optimal Aquifer Remediation Design'. Water Resources Research 31, 731-740.

33. Meza, J. C.: 1994, 'OPT++: An Object-Oriented Class Library for Nonlinear Optimization’. Technical Report SAND94-8225, Sandia National Laboratory.

34. Nelder, J. A. and R. Mead: 1965, 'A simplex method for function minimization'. Comput. J. 7, 308-313.

35. Reed, P.: 2003. private communication.

36. Reed, P., B. Minsker, and D. Goldberg: 2000, 'Designing a competent simple genetic algorithm for search and optimization'. Water Resources Research 36(12), 3757-3761.

37. Ritzel, B., J. Eheart, and S. Ranjithan: 1994a, 'Using genetic algorithms to solve a multiple objective groundwater pollution containment problem'. Water Resources Research 30(5), 1589-1604.

38. Ritzel, B. J., J. W. Eheart, and S. Ranjithan: 1994b, 'Using Genetic Algorithms to Solve a Multiple Objective Groundwater Pollution Containment Problem'. Water Resources Research 30(5), 1589-1603.

39. Rizzo, D. M. and D. E. Dougherty: 1996, 'Design optimization for multiple management period groundwater remediation'. Water Resources Research 32(8), 2549-2561.

40. Rogers, L. L. and F. U. Dowla: 1994, 'Optimization of Groundwater Remediation Using Artificial Neural Networks with Parallel Solute Transport Modeling'. Water Resources Research 30(2), 457-481.

41. Shanno, D. F.: 1970, 'Conditioning of quasi-Newton methods for function minimization'. Math. Comp. 24, 647-657.

42. Stoneking, D., G. Bilbro, R. Trew, P. Gilmore, and C. T. Kelley: 1992, 'Yield Optimization Using a GaAs Process Simulator Coupled to a Physical Device Model'. IEEE Transactions on Microwave Theory and Techniques 40, 1353-1363. 
43. Torczon, V.: 1989, 'Multidirectional Search'. Ph.D. thesis, Rice University, Houston, Texas.

44. Watkins Jr, D. and D. McKinney: 1998, 'Decomposition methods for water resources optimization models with fixed costs'. Advances in Water Resources 21(4), 261-324.

45. Zheng, C. and P. P. Wang: 1999, 'An integrated global and local optimization approach for remediation system design'. Water Resources Research 35(1), 137-148. 
katie.tex; 11/07/2003; 8:20; p.32 